\title{
Severe HELLP syndrome masquerading as thrombocytopenic thrombotic purpura: a case report
}

\author{
Cyril Mousseaux ${ }^{1 *}$ D, Bérangère S. Joly ${ }^{2}$, Inna Mohamadou', Romain Arrestier ${ }^{1}$, Alexandre Hertig ${ }^{1}$ and Cédric Rafat ${ }^{1}$
}

\begin{abstract}
Background: Thrombotic microangiopathies (TMAs) occurring in the postpartum period may be difficult to manage. They present as the combination of mechanical hemolytic anemia and consumption thrombocytopenia due to endothelial dysfunction. The cause of this endothelial aggression can be multiple: thrombocytopenic thrombotic purpura (TTP), HELLP syndrome, antiphospholipid syndrome, atypical hemolytic and uremic syndrome or acute fatty liver of pregnancy. TTP results from a severe deficiency of ADAMTS13, which is a protease cleaving specifically von Willebrand factor chiefly produced by liver cells. There are two main causes, the production of antiADAMTS13 auto-antibodies and, more rarely, a genetic deficiency in ADAMTS13. First-line treatment is based on plasma exchange. HELLP syndrome occurs in the third trimester of pregnancy usually in association with preeclampsia and represents a form of TMA characterized by damage to the sinusoidal capillaries of the liver. Prompt delivery is the main treatment. We present a case illustrating the challenges in discriminating between different postpartum TMAs, with a focus on the distinction between TTP and HELLP syndrome. Specifically, we highlight how acute liver failure (ALF) stemming from HELLP may lead to TTP with a spectacular response to plasma exchanges.
\end{abstract}

Case: A 28-year-old, $33+4$ weeks pregnant woman presented with severe preeclampsia complicated by ALF in the setting of partial liver necrosis, disseminated intravascular coagulation, microangiopathic hemolytic anemia and acute kidney injury. Greatly diminished levels of ADAMTS13 $(<5 \%)$ activity and neurological impairment suggested an initial diagnosis of thrombotic thrombocytopenic purpura (TTP). Therapeutic plasma exchange (TPE) was initiated and complete renal, neurological, hematological and hepatic recovery was observed. Secondary TTP induced by ALF due to HELLP syndrome was the final diagnosis.

Conclusion: Our case addresses the overlapping nature of postpartum TMAs and raises the possibility that HELLPinduced ALF may constitute an additional mechanism resulting in TTP, thereby opening a possible indication for TPE.

Keywords: Acute liver failure, HELLP syndrome, Therapeutic plasma exchange, Thrombotic microangiopathy, Thrombotic thrombocytopenic purpura, Case report

\footnotetext{
* Correspondence: mousseaux.cyril@gmail.com

'Department of Nephrology, CHU Tenon, Assistance Publique-Hopitaux de Paris, Paris, France

Full list of author information is available at the end of the article
}

(C) The Author(s). 2020 Open Access This article is licensed under a Creative Commons Attribution 4.0 International License, which permits use, sharing, adaptation, distribution and reproduction in any medium or format, as long as you give appropriate credit to the original author(s) and the source, provide a link to the Creative Commons licence, and indicate if changes were made. The images or other third party material in this article are included in the article's Creative Commons licence, unless indicated otherwise in a credit line to the material. If material is not included in the article's Creative Commons licence and your intended use is not permitted by statutory regulation or exceeds the permitted use, you will need to obtain permission directly from the copyright holder. To view a copy of this licence, visit http://creativecommons.org/licenses/by/4.0/ The Creative Commons Public Domain Dedication waiver (http://creativecommons.org/publicdomain/zero/1.0/) applies to the data made available in this article, unless otherwise stated in a credit line to the data. 


\section{Background}

Postpartum thrombotic microangiopathy (TMA) clusters multiple clinical entities with largely overlapping clinical presentation albeit with distinct physiopathology and requiring specific management. They include thrombocytopenic purpura (TTP), HELLP syndrome, antiphospholipid syndrome, atypical hemolytic and uremic syndrome or acute fatty liver of pregnancy. TTP is a rare and lifethreatening TMA characterized by a severe deficit in ADAMTS13 (disintegrin and metalloprotease with thrombospondin type 1 repeats, member 13) [1] either of antiADAMTS13 autoimmune etiology or genetic mutation of ADAMTS13. During pregnancy, TTP occurs primarily during the second and third semesters and genetic forms are more prevalent. Therapeutic plasma exchange (TPE) and corticosteroids form the mainstay of first line therapeutic management which may be complemented by rituximab and caplacizumab in refractory cases [2]. In case of concurrent preeclampsia, the favored diagnosis is HELLP syndrome which consists of peripheral signs of TMA associated with liver involvement (mainly liver cytolysis) and renal injury. In most situations, delivery and a supportive treatment prove to be curative. Exceptionally, HELLP syndrome may result in liver necrosis, and in fine acute liver failure (ALF). Herein, we present the case of a preeclamptic patient who developed liver necrosis following HELLP syndrome that was practically indistinguishable from TTP but which was successfully treated with TPE.

\section{Case presentation}

A 28-year-old woman was admitted for severe preeclampsia at $33+4$ weeks. It was her fifth pregnancy. None of her previous pregnancies had been complicated by preeclampsia. Her foetus presented with severe intrauterine growth restriction (estimated foetal weight $<3$ rd percentile).

Her initial physical examination was remarkable for hypertension and a dipstick proteinuria $(2+)$. She had no neurological symptoms.

Initial laboratory investigations (Table 1) revealed mild renal impairment, hyperleukocytosis, mild anemia without thrombocytopenia. Coagulation assays were unremarkable.

Two days after admission, at $33+6$ weeks, she presented with epigastric pain and her foetus was bradycardic ( 80 beats per minute). An emergency Cesarean section was performed during which a large retroplacental hematoma was uncovered. She delivered a $1400 \mathrm{~g}$ healthy baby boy. Postpartum hemorrhage (1.2 L) occurred immediately prompting the administration of $3 \mathrm{~L}$ of crystalloid fluids, and $1 \mathrm{~g}$ of tranexamic acid. In order to prevent eclampsia, treatment with magnesium sulfate was initiated.
Due to severe preeclampsia and anuria following the Cesarean section, she was transferred to the intensive care unit (ICU) for monitoring.

Physical examination revealed no fever $\left(37.7^{\circ} \mathrm{C}\right)$, controlled blood pressure $(138 / 84 \mathrm{mmHg}$ under $4 \mathrm{~g}$ per hour of intravenous nicardipine) and no tachycardia (97 beats per minute). Neurologic examination was remarkable for drowsiness (Glasgow score $=13$ ) with no evidence of focal neurological signs and normal osteotendinous reflexes. Epigastric pain was persistent.

Immediately after surgery, hemostasis disorders consistent with disseminated intravascular coagulation (DIC) appeared (Table 1) with an International Society on Thrombosis and Hemostasis score of seven, prompting the administration of five units of red blood cells, three units of fresh frozen plasma, and $3 \mathrm{~g}$ of fibrinogen. At this point, prothrombin time fell to $46 \%$, platelets and fibrinogen decreased to 23.000 and $1.92 \mathrm{~g} / \mathrm{L}$ respectively, and D-dimer value was above $20.000 \mu \mathrm{g} / \mathrm{L}$.

Signs of HELLP syndrome were present, as defined by severe cytolysis, thrombocytopenia and microangiopathic hemolytic anemia (Table 1). ALF developed, as defined by grade 3 encephalopathy, and a MELD score of 32 . Blood glucose test remained normal, but a peak lactate level of $7 \mathrm{mmol} / \mathrm{L}$ was observed on day 2. Simultaneously, an acute kidney injury (KDIGO stage 3) occurred (Table 1) requiring hemodialysis (overload and anuria). Finally, empiric antibiotic therapy with tazocillin was started due to suspected sepsis with fever plateau at 38.5.

Four days following delivery, the patient was referred to our renal ICU due to suspected TTP. The neurological examination was unchanged with persistent drowsiness. A brain magnetic resonance imaging (MRI) was deemed normal. Abdominal MRI showed heterogeneous high signal intensity on $\mathrm{T} 1$ and $\mathrm{T} 2$-weighted images in the posterior right lobe of the liver suggestive of liver necrosis (Fig. 1a, b, c).

In the hypothesis of a coincident TTP, methylprednisolone $(1 \mathrm{mg} / \mathrm{kg})$ and daily TPE with $100 \%$ frozen plasma units were initiated. By the fourth TPE, liver function and platelet count were restored to normal values (Fig. 1d). TPE was thereafter gradually tapered off with a total of 11 sessions (8 daily sessions, then two sessions spaced $48 \mathrm{~h}$ apart and one last session 4 days later).

As part of the diagnostic work-up, ADAMTS13 activity was found to be undetectable, yet anti-ADAMTS13 auto-antibodies were not found. Corticosteroids were stopped as soon as ADAMTS13 auto-antibody negativity was obtained (total duration of 9 days). A kidney biopsy demonstrated severe acute tubular necrosis but no signs of cortical necrosis or TMA. Atypical hemolytic and uremic syndrome was excluded from renal biopsy and 
Table 1 Laboratory investigations prior to Cesarean section to the intensive care unit (ICU) and to our unit

\begin{tabular}{|c|c|c|c|c|}
\hline Variable & Normal range & Prior to Cesarean & Admission to the ICU & Admission to our unit \\
\hline \multicolumn{5}{|l|}{ Hematology and coagulation assays } \\
\hline Hemoglobin ( $g / d L)$ & $12-16$ & 11.7 & 12.6 & 6 \\
\hline White cell count (per $\mu L$ ) & $4500-10,000$ & 21,000 & 27,600 & 30,000 \\
\hline \multicolumn{5}{|l|}{ Differential count (\%) } \\
\hline Neutrophils & $40-70$ & 76 & 76.7 & 83 \\
\hline Others & $30-70$ & & 24.3 & 17 \\
\hline Platelet count (per $\mu L$ ) & $150,000-400,000$ & 160,000 & 155,000 & 53,000 \\
\hline Fibrinogen (g/liter) & $2-6$ & 2.41 & 2 & 4.59 \\
\hline Schizocytes (\%) & $<1$ & 0 & Not specified & 2 \\
\hline Prothrombin time (\%) & $70-110$ & 85 & 61 & 58 \\
\hline Factor $V(\%)$ & $70-120$ & 75 & 24 & 70 \\
\hline Activated partial thromboplastin time ratio & $0.8-1.2$ & 1.2 & 1.21 & 1.31 \\
\hline D-dimers & $<500$ & & $>20,000$ & \\
\hline \multicolumn{5}{|l|}{ Renal analysis } \\
\hline Creatinine (mg/dL) & $0.6-1.5$ & 1.1 & 1.56 & 3.7 \\
\hline Blood urea nitrogen (mg/dL) & $8-25$ & & 14 & 38.8 \\
\hline \multicolumn{5}{|l|}{ Hepatic analysis } \\
\hline Albumin $(g / L)$ & $35-50$ & & 25 & 21 \\
\hline Lactate dehydrogenase (U/L) & $125-250$ & & 5000 & 7820 \\
\hline Haptoglobin $(g / L)$ & $0.2-0.4$ & & 0.2 & $<0.08$ \\
\hline Aspartate aminotransferase (U/L) & $<35$ & & Hemolysis & 1749 \\
\hline Alanine aminotransferase $(U / L)$ & $<45$ & & 500 & 833 \\
\hline Alkaline phosphatase (U/L) & $<115$ & & 229 & 283 \\
\hline Gamma glutamyl transpeptidase (U/L) & $<45$ & & 168 & 212 \\
\hline Bilirubin total/conjugate (mg/dL) & $0.3-1.9 / 0-0.3$ & & $13.7 / 8.9$ & $40 / 28.2$ \\
\hline Arterial lactate (mmol/L) & $0-2$ & & 1.9 & 2.2 \\
\hline \multicolumn{5}{|l|}{ TMA exploration } \\
\hline ADAMTS13 activity (\%) & $50-150$ & & & $<5 \%$ \\
\hline IgG anti-ADAMTS13 & & & & Negative \\
\hline Coombs test & Negative & & Negative & \\
\hline HIV test & & & Negative & \\
\hline C3 $(g / L)$ & $0.9-1.8$ & & 0.56 & \\
\hline C4 $(g / L)$ & $0.1-0.4$ & & 0.06 & \\
\hline Antinuclear and anti-DNA antibodies & & & Negative & \\
\hline Factor $H(\%)$ & $65-140$ & & 106 & \\
\hline Factor I (\%) & $70-130$ & & 125 & \\
\hline Factor H autoantibody & & & Negative & \\
\hline Antiphospholipid antibodies $^{\mathrm{a}}$ & & & Negative & \\
\hline
\end{tabular}

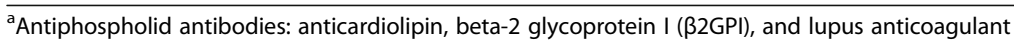

alternate complement pathway explorations, including genetic analysis (Table 1). Acute fatty liver of pregnancy was ruled out given the presence of hemolysis, and the absence of hypoglycemia and hyperechogenicity on liver ultrasound. Antiphospholipid antibody syndrome was also ruled out.
ADAMTS13 activity rose to 17 and 24\%, 4 and 7 days, respectively, after discontinuation of TPE. Genetic analysis of ADAMTS13 did not reveal any sequence variation. Both antibody-mediated TTP and congenital TTP were thus excluded. On hospital discharge (27 days after delivery) the patient had recovered complete liver and kidney function. 


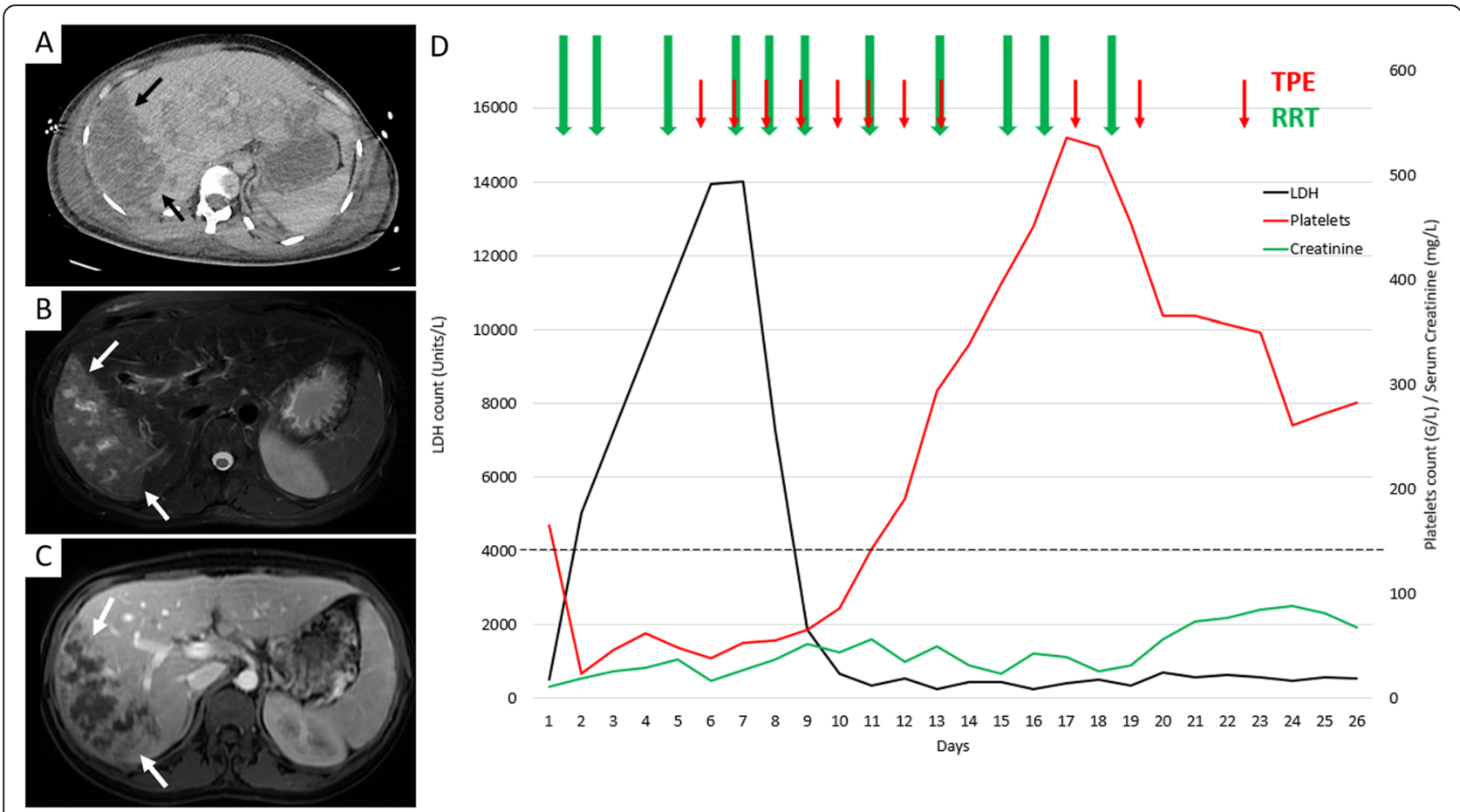

Fig. 1 Imaging and temporal trends of lactate dehydrogenase (LDH) levels and platelets following delivery. Computerized Tomography scan (a) with axial reconstruction shows lack of perfusion of the posterior sector of the liver (black arrow). Magnetic Resonance Imaging with T2 sequence (b) and T1 sequence with gadolinium injection (c) show high signal T2 of the posterior sector of the liver related to necrosis (white arrows) and lack of enhancement after gadolinium injection (white arrows) related to the infarction of the hepatic parenchyma. Therapeutic plasma exchange (TPE, red arrows) was initiated at day 5 following delivery and renal replacement therapy (RRT, green arrows) at day 1. Serum lactate dehydrogenase (LDH, black line), platelet count (red line) and serum creatinine (green line, mg/L) are given on the ordinate. Day 1 is the day of delivery. The dotted line is the standard for platelets

\section{Discussion and conclusion}

Our case demonstrates that ALF caused by a HELLP syndrome may be severe enough to induce severe ADAMTS13 deficiency and elicit the full range of clinical symptoms associated with TTP. Akin to the autoimmune and congenital forms of the condition, this cause of secondary TTP is also amenable to TPE. Yet its timely recognition and differentiation from the canonical forms of TTP is essential to avoid unnecessary and potentially harmful treatments such as immunosuppressive therapy, a mainstay in the auto-immune form of the disease, or repeated prophylactic TPE, as considered in some cases of congenital TTP.

The typical course of platelets during a HELLP syndrome has been previously described [3]. Here, the persistence of TMA features, along with neurological impairment, encouraged us to prioritize a diagnosis of TTP and to initiate TPE.

Central to these investigations is the assessment of ADAMTS13 activity since severe functional deficiency in ADAMTS13 $(<10 \%)$ serves as the indisputable pathophysiological basis for TTP. Reciprocally, severe ADAMTS13 deficiency is held to be the only specific, if not quasi-pathognomonic, diagnostic marker of the disease [1].
TTP may be caused by either IgG auto-antibodies directed against ADAMTS13 or by a recessively inherited bi-allelic mutation of the ADAMTS13 gene. In adulthood, this latter mechanism is more likely to occur during pregnancy than in other settings [4].

This case contends that a third mechanism may be involved: the severity of liver failure, as evidenced by the lowered levels of factor V (30\%) along with partial liver necrosis. Because of the dual blood supply of the liver, liver necrosis is an exceptional complication of HELLP syndrome [5]. A retrospective analysis derived from the UNOS databank yielded only eight cases of fulminant liver failure from HELLP syndrome requiring liver transplantation [6].

Therefore, the diagnosis hinged on whether the patient presented with genuine TTP, or whether HELLP syndrome may have precipitated the collapse in ADAMTS13 levels. The fact that the flare of TMA was observed postpartum (in contrast with what is typically observed in patients with primary TTP) [7], and that the context was of a preeclampsia-related liver injury, argued for such a scenario. Moreover, TTP is not usually associated with fetal abnormality because placenta function is preserved. 
In patients with HELLP syndrome, low levels of ADAMTS13 have indeed been reported but, to our knowledge, never in the critical range $(<10 \%)$ where TTP should be regarded as a superimposed diagnosis. In fact, in one series which specifically focused on ADAMTS13 assessment in patients with HELLP syndrome, the lowest recorded ADAMTS13 level was 12\% [8]. In two other case series, two patients diagnosed initially with HELLP syndrome did exhibit ADAMTS13 levels below $5 \%$, but in both cases the final diagnosis was later revised to congenital TTP $[9,10]$.

Liver function integrity is a prerequisite for normal ADAMTS13 production [11]. Accordingly, ADAMTS13 plasma levels have been shown to be inversely correlated to the severity of liver injury both in the chronic setting of liver cirrhosis and in ALF [12]. In various conditions other than HELLP syndrome, ALF has been shown to be associated with greatly diminished ADAMTS13 levels, below the threshold required for TTP diagnosis.

Another prominent feature in this case was the occurrence of severe DIC. DIC has been proposed as a possible cause of ADAMTS13 [13] consumption although some authors have suggested that sepsis-induced inflammation may in fact be the key trigger in reducing the levels of ADAMTS13 [14].

Rather than HELLP syndrome per se, it is therefore plausible that the direct and indirect clinical complications of HELLP syndrome, most importantly ALF, with the possible contribution of DIC and sepsis, may have coincided to reduce ADAMTS13 levels to such an extent that TTP was genuinely manifested, especially against the backdrop of neurological impairment. However, the absence of antibodies against ADAMTS13, as well as the rebound of ADAMTS13 levels following recovery and the absence of genetic abnormalities in the ADAMTS13 gene prompted us to retain the final diagnosis of secondary TTP resulting from HELLP-induced ALF. Given the initial hypothesis of severe TTP, TPE was initiated. Within $24 \mathrm{~h}$ there was such an extensive improvement in the patient's neurological status as well as in LDH and platelet levels (Fig. 1d) that these were originally interpreted as further evidence supporting this diagnosis. TPE has previously been investigated in the context of HELLP syndrome with several studies reporting accelerated recovery following TPE initiation [15]. However, most studies were observational and conducted at a time when ADAMTS13 assessment was not readily available, thus suggesting a possible case mix effect. Likewise, the indication of TPE has also been examined in ALF with encouraging results [16]. Finally, although TPE was originally believed to be possibly detrimental in the setting of sepsis, it has been shown recently that such patients may in fact safely benefit from TPE, and even more so when DIC is present [17].
Although seemingly disparate, TPE plays a similar role in these conditions, namely to replenish the levels of ADAMTS13, to correct coagulopathy, provided that fresh frozen plasma is used, and perhaps to mitigate the inflammatory cascade. Remarkably, the patient encapsulated all these features.

Taken together, this case should inspire clinicians to move away from the common heuristic whereby a satisfactory response to TPE provides indisputable evidence in favor of auto-immune or congenital TTP. More importantly, it begs the question as to whether plasma exchange may benefit a subset of patients with severe HELLP syndrome with markedly diminished levels of ADAMTS13.

\section{Abbreviations}

ADAMTS13: Disintegrin and metalloprotease with thrombospondin type 1 repeats, member 13; ALF: Acute liver failure; DIC: Disseminated intravascular coagulation; MRI: Magnetic resonance imaging; TMA: Thrombotic microangiopathies; TPE: Therapeutic plasma exchange;

TTP: Thrombocytopenic thrombotic purpura

\section{Acknowledgements}

We would like to acknowledge the Department of Obstetrics, and the ICU of Versailles Hospital for the initial management of this patient. Dr. Virginie Cadel-Goudard, obstetrician, was involved in the initial gynecological management and the Cesarean section. Dr. Antoine Gros, intensivist, was involved in initial intensive care management. We would like to acknowledge the complementary investigation performed by Dr. Veronique FremeauxBacchi in the Department of Immunology, Hôpital Européen Georges Pompidou, Paris.

\section{Authors' contributions}

$C M, B J, I M, R A, A H, C R$ were involved in diagnosis, management, and followup at Tenon Hospital ICU. CM, AH, CR were responsible for research concept and study design. Data acquisition was done by CM. ADAMTS13 analyses were performed by BJ. CM, AH, CR contributed substantially to the literature review, drafting of the initial manuscript of the case report, critical revision and its final version. All authors have contributed, read and approved the final and revised manuscript.

\section{Funding}

No support/funding was claimed or received by any author from any source for this work.

\section{Availability of data and materials}

If required, the relevant material can be provided by corresponding author on reasonable request.

\section{Ethics approval and consent to participate Not applicable.}

\section{Consent for publication}

Written informed consent for publication of clinical details was obtained from the patient. A copy of the consent form is available for review by the Editor of this journal.

\section{Competing interests}

$\mathrm{AH}$ is an Editorial Board Member of BMC Nephrology.

\section{Author details}

'Department of Nephrology, CHU Tenon, Assistance Publique-Hopitaux de Paris, Paris, France. ${ }^{2}$ Hematology Laboratory, French Reference Center for Thrombotic Microangiopathies, Hôpital Lariboisière, Assistance

Publique-Hôpitaux de Paris, Paris, France. 
Received: 6 November 2019 Accepted: 25 May 2020

Published online: 29 May 2020

\section{References}

1. Joly BS, Coppo P, Veyradier A. Thrombotic thrombocytopenic purpura. Blood. 2017;129(21):2836-46.

2. Scully M, Thomas M, Underwood M, Watson H, Langley K, Camilleri RS, et al. Thrombotic thrombocytopenic purpura and pregnancy: presentation, management, and subsequent pregnancy outcomes. Blood. 2014;124(2):211-9.

3. Wallace K, Martin JN, Tam Tam K, Wallukat G, Dechend R, Lamarca B, et al. Seeking the mechanism(s) of action for corticosteroids in HELLP syndrome: SMASH study. Am J Obstet Gynecol. 2013;208(5):380.e1-8.

4. Moatti-Cohen M, Garrec C, Wolf M, Boisseau P, Galicier L, Azoulay E, et al. Unexpected frequency of Upshaw-Schulman syndrome in pregnancy-onset thrombotic thrombocytopenic purpura. Blood. 2012;119(24):5888-97.

5. Yoshihara M, Mayama M, Ukai M, Tano S, Kishigami Y, Oguchi H. Fulminant liver failure resulting from massive hepatic infarction associated with hemolysis, elevated liver enzymes, and low platelets syndrome. J Obstet Gynaecol Res. 2016;42(10):1375-8.

6. Shames BD, Fernandez LA, Sollinger HW, Chin LT, D'Alessandro AM, Knechtle SJ, et al. Liver transplantation for HELLP syndrome. Liver Transplant Off Publ Am Assoc Study Liver. Dis Int Liver Transplant Soc. 2005;11(2):224-8.

7. Fakhouri F, Roumenina L, Provot F, Sallée M, Caillard S, Couzi L, et al. Pregnancy-associated hemolytic uremic syndrome revisited in the era of complement gene mutations. J Am Soc Nephrol JASN. 2010;21(5):859-67.

8. Lattuada A, Rossi E, Calzarossa C, Candolfi R, Mannucci PM. Mild to moderate reduction of a von Willebrand factor cleaving protease (ADAMTS13) in pregnant women with HELLP microangiopathic syndrome. Haematologica. 2003;88(9):1029-34.

9. Sabau L, Terriou L, Provot F, Fourrier F, Roumier C, Caron C, et al. Are there any additional mechanisms for haemolysis in HELLP syndrome? Thromb Res. 2016;142:40-3.

10. Pourrat $\mathrm{O}$, Coudroy R, Pierre F. ADAMTS13 deficiency in severe postpartum HELLP syndrome. Br J Haematol. 2013;163(3):409-10.

11. Uemura M, Tatsumi K, Matsumoto M, Fujimoto M, Matsuyama T, Ishikawa M et al. Localization of ADAMTS13 to the stellate cells of human liver. Blood. 2005;106(3):922-4.

12. Hugenholtz GCG, Adelmeijer J, Meijers JCM, Porte RJ, Stravitz RT, Lisman T. An unbalance between von Willebrand factor and ADAMTS13 in acute liver failure: implications for hemostasis and clinical outcome. Hepatol Baltim Md. 2013;58(2):752-61.

13. Ono T, Mimuro J, Madoiwa S, Soejima K, Kashiwakura Y, Ishiwata A, et al. Severe secondary deficiency of von Willebrand factor-cleaving protease (ADAMTS13) in patients with sepsis-induced disseminated intravascular coagulation: its correlation with development of renal failure. Blood. 2006; 107(2):528-34.

14. Peigne V, Azoulay E, Coquet I, Mariotte E, Darmon M, Legendre P, et al. The prognostic value of ADAMTS13 (a disintegrin and metalloprotease with thrombospondin type 1 repeats, member 13) deficiency in septic shock patients involves interleukin- 6 and is not dependent on disseminated intravascular coagulation. Crit Care Lond Engl. 2013;17(6):R273.

15. Erkurt MA, Berber I, Berktas HB, Kuku I, Kaya E, Koroglu M, et al. A life-saving therapy in class I HELLP syndrome: therapeutic plasma exchange. Transfus Apher Sci Off J World Apher Assoc Off J Eur Soc Haemapheresis. 2015;52(2):194-8.

16. Larsen FS, Schmidt LE, Bernsmeier C, Rasmussen A, Isoniemi H, Patel VC, et al. High-volume plasma exchange in patients with acute liver failure: an open randomised controlled trial. J Hepatol. 2016;64(1):69-78.

17. Putzu A, Schorer R, Lopez-Delgado JC, Cassina T, Landoni G. Blood purification and mortality in Sepsis and septic ShockA systematic review and meta-analysis of randomized trials. Anesthesiol J Am Soc Anesthesiol. 2019;131(3):580-93.

\section{Publisher's Note}

Springer Nature remains neutral with regard to jurisdictional claims in published maps and institutional affiliations.

Ready to submit your research? Choose BMC and benefit from:
- fast, convenient online submission
- thorough peer review by experienced researchers in your field
- rapid publication on acceptance
- support for research data, including large and complex data types
- gold Open Access which fosters wider collaboration and increased citations
- maximum visibility for your research: over 100M website views per year
At BMC, research is always in progress.
Learn more biomedcentral.com/submissions

